\title{
ACCESSIBILIDAD VIARIA Y DESARROLLO TERRITORIAL EN LA ESPAÑA PENINSULAR: UNA PROPUESTA DE ANÁLISIS*
}

\author{
Nicoletta Nastagi Mattoni \\ Departamento de Ciencias Económicas y Empresariales, Facultad de Economía \\ Universidad de Florencia * \\ Curtatone 1, 50123 Florencia - E-mail: dipscien@cce.unifi.it
}

\begin{abstract}
Resumen: En este trabajo se analizan los aspectos más significativos de la red de carreteras española, poniéndose especial énfasis en la reciente evolución. Particularmente se propone un análisis sobre las relaciones existentes entre el desarrollo de la red viaria y el desarrollo económico y social del territorio donde la misma está inscrita. Tal propuesta se articula por medio de la cuantificación del concepto de accesibilidad y su sucesiva asociación a la magnitud demográfica.
\end{abstract}

Palabras clave: España, red de carreteras, accesibilidad.

\begin{abstract}
This study analyzes the more significant aspects of the development of the Spanish road network, giving emphasis to the recent evolution. In particular, it offers an analysis of the relationship that appears between the development of the various Spanish networks and the development of the social economy of the territory within the same network developments. This proposal is articolated through the quantifing of the concept of accessibility and on the eventual association of the demografic expansivness.
\end{abstract}

Key Words: Spain, road network, accessibility.

\section{Introducción}

La red de carreteras española ha sufrido una importante evolución durante los últimos veinte años, caracterizada por un gran crecimiento económico y un notable incremento de las exigencias de movilidad internas e internacionales. En este sentido el desarrollo viario ha permitido a España reducir el déficit que su patrimonio viario

\footnotetext{
* Recibido: 1-2-2002 Aceptado: 7-4-2003
} 
denunciaba respecto a los otros países europeos a comienzos de los años 80, desde un punto de vista tanto cuantitativo como cualitativo (Ministerio de Fomento, 1998).

La bibliografía dedicada al análisis de las infraestructuras viarias y a la relación que existe entre éstas y al desarrollo de las áreas geográficas donde las mismas se desarrollan es abundante (Bihel, 1986 y 1988; Sáenz de Buruaga, 1989; Pérez Esparcia, 1988; Moreno Jiménez, 1985; Capineri, Tinacci y otros, 1996). En general se afirma que la red de carreteras, como sistema organizado de carreteras, genera una reducción de las distancias y un incremento de la accesibilidad en términos de reducción de los costes de viaje con importantes consecuencias económicas y sociales (Gutiérrez Puebla, 1998). En el ámbito comunitario se concluye que las infraestructuras de transporte contribuyen a reforzar la cohesión económica entre los países de la Comunidad y que los beneficios de integración están influidos por la existencia de una red de infraestructuras flexibles y capaces de ofrecer conexiones eficientes entre las regiones (ECC, 1989 y 1995).

La propuesta de análisis expuesta en este trabajo se desarrolla sobre una metodología de tipo descriptivo y está centrada en la interpretación del concepto de accesibilidad y en la relación con la magnitud demográfica. La aplicación metodológica se basa en el concepto de accesibilidad potencial (MOPU, 1984) y en una sucesiva elaboración a través el uso de algunos instrumentos estadísticos. En particular se ha desarrollado una reelaboración del mismo modelo de accesibilidad junto a la utilización de algunos conceptos de la teoría de grafos. Es cierto, sin embargo, que la metodología que hemos usado es sólo una de las muchas y experimentadas en este ámbito de estudio.

El trabajo está estructurado del modo siguiente: en la primera sección (capítulo 2) se describen los trazos más significativos de la red viaria española centrándose en la Red de Gran Capacidad (RGC) en cuanto que tales informaciones representan elementos indispensables para argumentar algunos de los principales resultados añadidos sucesivamente.

En la segunda sección del trabajo, compuesta por el capítulo 3 y siguientes, viene expuesta nuestra propuesta de análisis y luego aplicada a diez ciudades de la red de carreteras. El número de ciudades que ha sido considerado puede parecer insuficiente al fin de sacar conclusiones sobre el estudio propuesto. En relación a este aspecto es oportuno precisar que tal decisión está en función de varias razones; en particular, este articulo representa una sistesís de un tabajo desarrollado sobre la geografía de España y sobre el tema de sus infraestructuras de transporte. De modo que a la hora de completar el mismo se ha considerado interesante realizar un estudio de tipo metodológico sobre la evolución de las carreteras y su relación con el desarrollo demográfico. La metodología creada ha sido luego aplicada tenendo en cuenta algunos nudos de la misma red de carreteras con el fin de ofrecer un ejemplo sobre su concreta aplicación y utilidad. 


\section{La red de carreteras española y su evolución reciente}

Las carreteras españolas ${ }^{2}$ se pueden clasificar en relación con el ente público que las gestiona así como en relación a sus características técnico-funcionales. En base al primer criterio se distinguen en España tres categorías de carreteras que respectivamente forman la red nacional de carreteras (RIGE: Red de Interés General del Estado), la red de carreteras autonómica y la red de carreteras local (provincial y comunitaria). En relación a las intervenciones legislativas sucesivas a la Constitución, han sido oficialmente reconocidas como carreteras que forman parte de la RIGE aquellas carreteras integradas en un itinerario de interés general que cumplen al menos uno de los siguientes requisitos: representar trazos de los principales itinerarios del tráfico u ofrecer el acceso a un puerto o aeropuerto de interés general; o, finalmente conectar los principales centros urbanos del territorio formando una red continua como soporte del tráfico de largo recorrido.

Según el segundo criterio, el técnico-funcional, las carreteras españolas se clasifican en autopistas, autovías y carreteras convencionales: las dos primeras tipologías, junto a las carreteras de circunvalación de centros urbanos (con cuatro o más carriles) representan la Red de Gran Capacidad (RGC); la tercera tipología está constituida por las genéricas carreteras interurbanas.

El reciente desarrollo de la red nacional española (RIGE) ha seguido las directivas del Plan de Carreteras 1984-1991 y del Plan de las Infraestructuras 1993-2007. El Plan de Carreteras ha enderezado y sostenido el desarrollo viario de España durante los años 80 , permitiendo una mejora del equilibrio económico y social entre las distintas regiones y una satisfacción parcial de las exigencias modernas de movilidad. A partir del 1993 y según el Plan de las Infraestructuras, la red viaria se desarrolla no sólo para satisfacer las exigencias actuales de movilidad, sino también para aquellas previstas para el futuro favoreciendo la coordinación entre las diversas modalidades de transporte. De otro modo, el papel de la red viaria nacional es el de garantizar un mejor nivel de accesibilidad tanto para el territorio como para los centros urbanos menores.

\subsection{La Red de Gran Capacidad (RGC)}

Antes del 1984 la dotación que comprende toda la red viaria española era suficiente en extensión pero precaria desde el punto de vista técnico-cualitativo. En particular, la red principal del país (RGC) evidenciaba una rígida estructura radial escasa de conexiones transversales, de tal forma que incidía negativamente sobre el nivel de accesibilidad de buena parte del territorio. 
A finales de 1993, gracias a la ejecución casi por completo (92'8\%) del Plan de Carreteras la RGC llegó a tener una longitud de $6.480 \mathrm{~km}$, acrecentándose una media de $464 \mathrm{~km}$ por año a partir del 1984. En este ámbito las autopistas con peaje han registrado un incremento bastante limitado (tan sólo $110 \mathrm{~km}$ entre el 1984 y el 1991) respecto al desarrollo observado en las autovías y en las autopistas sin peaje: $483 \mathrm{~km}$ en el $1984 \mathrm{y}$ $4550 \mathrm{~km}$ en el 1993. En base a los datos de 1998 la longitud global de la red de carreteras interurbanas alcanzaba los $163.273 \mathrm{~km}$, de los cuales el 14\%\% eran estatales, el $43 \% 4 \%$ pertenecían a las comunidades autónomas y el $42 \%$ a las provincias y ayuntamientos.

Gracias a tal desarrollo la actual estructura geométrica del RGC, que desde 1993 incluye también los trazos de carretera de conexión directa entre autopistas y autovias, no es estrictamente radial. Para describir su estructura geométrica es interesante evidenciar dos grandes áreas en el territorio peninsular con características muy diferentes la una respecto de la otra. Estas áreas son: el área central y el área costera (Figura 1)

En el área central la red de carreteras se desarrolla sobre seis ejes lineales, que parten del mismo origen, que es Madrid, nudo principal de la red española ${ }^{3}$. En este área, el grado de desarrollo en el diseño geométrico de la red respecto al originario de tipo radial, se observa sólo parcialmente por la construcción de un solo eje de conexión transversal que une las dos aristas orientadas hacia el norte (Madrid-La Coruña) y hacia el norte-este (Madrid-San Sebastián).

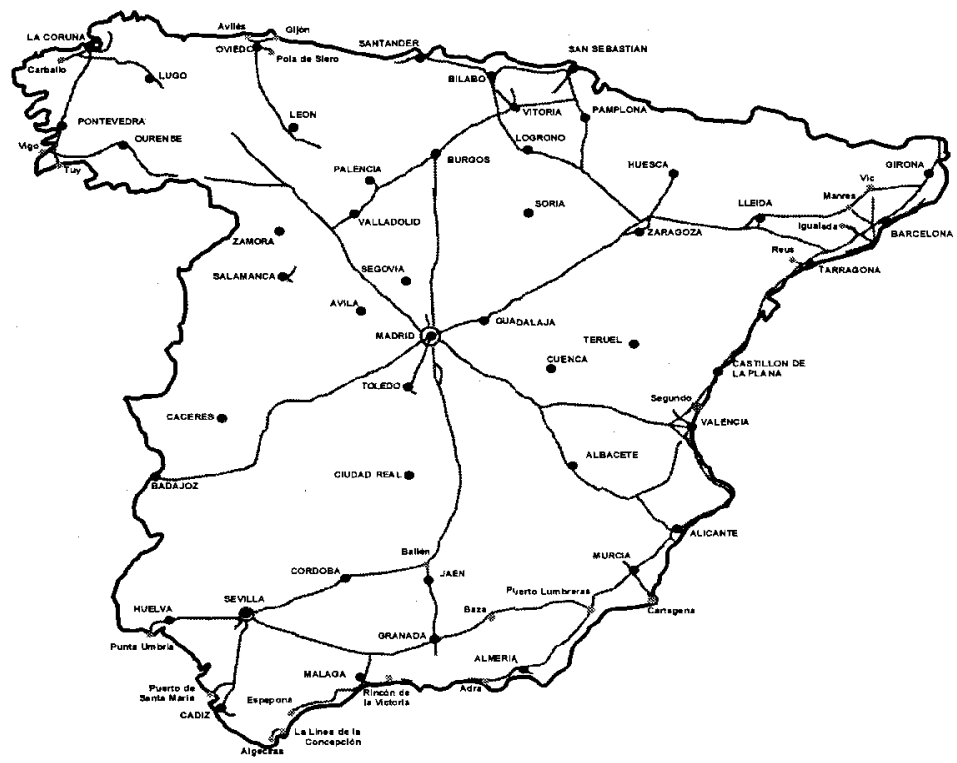

Figura 1. Red de Grande Capacidad (RGC) en la España peninsular (1998). 
En el área costera es posible reconocer una estructura geométrica de la red de tipo lineal. En particular a lo largo de la vertiente mediterránea el itinerario de la RGC es continuo desde de la frontera francesa hasta Adra (Almería), donde se interrumpe, para reiniciarse en el Rincón de la Victoria (Málaga) hasta Algeciras (Cádiz). En torno a los nudos de Barcelona y Valencia la red se hace más compleja dando origen a dos estructuras de corredor. Partiendo de Murcia hacía la región más occidental la estructura se convierte en más simple manifestando un estadio de desarrollo menor respecto al desarrollo observado en el tramo Barcelona Murcia. En el area costera septentrional es reducido el grado de continuidad por el itinerario Francia-Portugal ya que la RGC está, ya sea en extensión ya sea desde el punto de vista estructural, poco desarrollada. Una buena conexión és la que encontramos en el itinerario Santander-San Sebastián-Bilbao que se prolonga hacia el interior con dos arcos (Pamplona y Vitoria) en un único vértice conectado con Zaragoza. El nudo de Zaragoza tiene una importancia especial en el cuadro global de la RGC española en cuanto a que en él confluyen la circulación de los flujos hacia las principales áreas socio-económicas del país (País Vasco, Cataluña y Madrid).

La estructura de la RGC presentará una configuración más uniforme y compleja en función a las obras de reparto viario que se realizarán con la finalización del Plan de Infraestructuras 1993-2007. La red, de hecho, se enriquecerá con nuevos arcos de conexión entre los nudos del sistema, creándose así un sistema más evolucionado de organización jerárquica de los nudos y de las líneas.

\section{Grado de accesibilidad: una posible lectura}

Para valorar la accesibilidad que la red confiere en diversos puntos del territorio se ha utilizado el modelo de accesibilidad potencial. Tal modelo se estructura sobre la comparación de la relación existente entre tres tipologías de redes:

- la red real (de tiempo mínimo): representada por el sistema de itinerarios de carreteras por el cual el tiempo de recorrido entre cada par de nudos es el menor respecto a cada posible alternativa;

- la red potencial (de distancia mínima): formada del sistema de itinerarios de carreteras por el cual la distancia entre cada par de nudos es la menor respecto a cada posible alternativa;

- la red ideal o ficticia (de distancia en línea recta): formada del sistema de itinerarios por el cual la distancia entre cada par de nudos coincide con la longitud del segmento que los une. Esta red es utilizada para relativizar las variables referentes a la red real y potencial. 
Para cada pareja de nudos $i-j$ se considera la variable distancia $\left(D_{i j}\right)$, la variable tiempo de recorrido $\left(T_{i j}\right)$ y la velocidad de circulación $\left(V_{i j}\right)^{4}$. Los símbolos de las tres variables se refieren a la red real si no se utiliza ningún símbolo colocado al lado, mientras estén referidos a la red potencial y ficticia si van unido respectivamente con los símbolos (") y ( ). Para la red real y potencial la velocidad de recorrido en cada arco $i-j$ se ha igualado a la velocidad media de circulación a lo largo del arco considerado; y para la red ideal se ha asumido una misma velocidad en todos los arcos $i-j$ que la componen, e igual a la velocidad media de circulación en toda la red de carreteras considerada. La velocidad de circulación a la cual se hace referencia ha sido determinada por la Dirección General de Tráfico según la técnica del "vehículo flotante"s.

Gracias a la comparación entre la red real y la red potencial con la red ideal se puede medir y valorar la accesibilidad de los nudos del correspondiente sistema de carreteras y es posible hacer consideraciones sobre el desarrollo de la estructura de la red viaria del territorio tomado a examen.

En particular a través de la relación de las variables tiempo y distancia de los respectivos itinerarios con valor mínimo -real para el tiempo $T_{i j}$, potencial para la distancia $D_{i j^{-}}^{\prime}$ con las respectivas variables referidas al itinerario ideal $\widetilde{T}_{i j}$ y $\tilde{D}_{i j}$ se pueden construir dos clases de índices de un nudo $(i)$ respecto a otro $(j)$ :

Índice del tiempo real $i$-j: $I T_{i j}=\tilde{T}_{i j} / T_{i j}$

Índice de la distancia potencial $i-j: I D_{i j}=\widetilde{D}_{i j} / D_{i j}^{\prime}$

El resultado de tales índices está comprendido entre 0 y $+\infty$; el grado de accesibilidad, sea del tiempo real sea de la distancia potencial, es mayor conforme aumenta el resultado del relativo índice. Obviamente estas dos categorías de los indices están ligadas entre ellas. De hecho, considerada la relación del tiempo de recorrido $T_{i j}=D_{i j}$ $/ V_{i j}$ sigue:

$$
I T_{i j}=\frac{\widetilde{T}_{i j}}{T_{i j}}=\frac{\widetilde{D}_{i j}}{D_{i j}} \cdot \frac{V_{i j}}{\widetilde{V}}=I D_{i j} \cdot \frac{D_{i j}^{\prime}}{D_{i j}} \cdot \frac{V_{i j}}{\widetilde{V}} \sigma
$$

Siguiendo los planteamientos hasta ahora ilustrados, podemos construir un índice que mide el grado de accesibilidad del tiempo real de un nudo respecto a todos los otros nudos en el modo siguiente:

$$
\text { Índice del tiempo real para el nudo } i: \quad I T_{i}=\sum_{j \neq i} \widetilde{T}_{i j} / \sum_{j \neq i} T_{i j}
$$


El índice $I T_{i}$ construido mantiene una estructura análoga al elemental $I T_{i j}$; es de fácil interpretación, numeración y denominadores tienen un sentido bien definido en términos de distancia del nudo considerado respecto a todos los otros. También en este caso los resultados están comprendidos entre 0 y $+\infty$. Cuanto más alto es el resultado del índice, más alto es el nivel de accesibilidad del nudo $i$, desde y hacia los otros nudos de la red de carreteras, mientras la accesibilidad del nudo $i$ disminuye cuanto más se acerca a 0 el resultado del índice.

El índice introducido puede ser expresadó como media ponderada ${ }^{7}$ de los índices elementales $\left(I T_{i f}\right)$ de la forma siguiente:

$$
I T_{i}=\sum_{j \neq i} I T_{i j} w_{i j}
$$

donde la ponderación se establece a partir de de $W_{i j}=T_{i j} / \sum_{i j} T_{i j}$, el tiempo transcurrido al recorrer el tramo $i$ - $j$ respecto al tiempo total que pasa para llegar desde $i$ a cada uno de los otros nudos considerados.

Además es posible construir un índice que mide el grado de accesibilidad del tiempo real para toda la red, con una interpretación análoga a los precedentes. Índice del tiempo real de toda la red, o:

$$
I T=\sum_{i} \sum_{j \neq i} \widetilde{T}_{i j} / \sum_{i} \sum_{j \neq i} T_{i j}
$$

Esto mismo también puede ser expresado como media ponderada de los índices $I T_{i}$.

\section{La propuesta de análisis}

\subsection{Aplicaciones del concepto de accesibilidad presentado}

Después de haber expuesto el núcleo de la teoría de la accesibilidad potencial e introducido algunas modificaciones en el cálculo del índice del tiempo real, con el fin de realizar una más fácil lectura y aplicación, se ha calculado el mismo índice para diez nudos de la red para ejemplificar su utilización y proponer un posible análisis de la relación entre accesibilidad y desarrollo demográfico (Tabla 1).

Los diez nudos son representantes de algunas de las principales ciudades españolas, todas capital de provincia y centros de áreas metropolitanas. Con respecto a tales 
características en algunos casos han sido preferidas localidades ubicadas en la vertiente occidental de la península con respecto a otras ubicadas en el área oriental con el fin de tener una mayor cobertura del territorio peninsular español. Los valores de las variables $T, V$ y $D$ necesarias para el cálculo del indice, provienen de los datos disponibles en el Ministerio de Fomento; la velocidad media $(\tilde{V})$ de circulación para el año 1991 es de $78^{\prime} 02 \mathrm{~km} / \mathrm{h}$ y de $92^{\prime} 3 \mathrm{~km} / \mathrm{h}$ en el 1998 (Ministerio de Fomento, 1995 y 1999).

En la tabla 1 para cada nudo están recogidos los resultados del grado de accesibilidad real $\left(I T_{i}\right)$ y el respectivo numero de habitantes ${ }^{8}$. Es oportuno recordar, con el fin de una mejor lectura de los datos extraídos, que los mismos resultados consideran los datos relativos a sólo 10 nudos de la red y toman como referencia sólo las conexiones nacionales.

Tabla 1. Valores del índice de accesibilidad y número de habitantes. Años 1991 y 1998.

\begin{tabular}{|lcccr|}
\hline \multirow{2}{*}{ Nudos } & \multicolumn{2}{c}{1991} & \multicolumn{2}{c|}{1998} \\
\cline { 2 - 5 } & \multicolumn{1}{c}{$I T_{i}$} & $\mathrm{n}^{\circ}$ habitantes & \multicolumn{1}{c|}{$I T_{i}$} & $\mathrm{n}^{\circ}$ habitantes \\
\hline 1. Barcelona & 0,990 & 1.625 .542 & 1,044 & 1.505 .581 \\
2. Bilbao & 0,799 & 368.710 & 1,012 & 358.467 \\
3. Coruña (La) & 0,786 & 245.459 & 1,024 & 243.134 \\
4. Madrid & 0,899 & 2.909 .792 & 1,083 & 2.881 .506 \\
5. Málaga & 0,831 & 512.136 & 1,004 & 528.079 \\
6. Murcia & 0,913 & 318.838 & 1,087 & 349.040 \\
7. Oviedo & 0,810 & 194.919 & 1,023 & 199.549 \\
8. Sevilla & 0,803 & 659.126 & 1,004 & 701.927 \\
9. Valencia & 0,853 & 752.909 & 1,074 & 739.419 \\
10. Valladolid & 0,849 & 328.365 & 1,046 & 319.946 \\
\hline
\end{tabular}

Fuente: Elaboración propria a partir de datos del Ministerio de Fomento.

Se observa que el índice ha mejorado para todos los nudos considerados por nosotros aunque en diferente medida según los nudos. Un mejoramiento muy consistente se registra en los nudos localizados a lo largo de la costa septentrional (La Coruña, Oviedo y Bilbao) y en los dos nudos inscritos en el área sur-occidental (Sevilla y Málaga). Una variación en los mismos términos se observa también en el nudo de Valladolid. A la luz de las fuertes inversiones en el sector viario, que han llevado a tener una buena estructura de la RGC a lo largo del arco mediterráneo, es interesante destacar cómo Valencia representa el nudo con mayor incremento del grado de accesibilidad. Eso ha permitido a tal nudo formar parte, junto a Madrid y Murcia, del grupo de los nudos del sistema más altamente accesible en 1998 (ver capítulo 2.1). 
Con el fin de tener una visión sobre la accesibilidad que existe entre cada pareja de nudos, se ha calculado el índice de tiempo real para cada pareja de nudos $\left(I T_{i p}\right)$ para el año 1998. A través de la figura 2, cartograma realizado con los mismos resultados, se puede comprobar que todos los nudos situados sobre la costa septentrional de la península tienen un grado de accesibilidad real desarrollado con el nudo Madrid. El grado de accesibilidad entre el Norte-Sur está favorecido por la posición central del nudo de Madrid que representa el nudo con un grado de accesibilidad real que no es inferior a 1 con todos los otros nudos, exceptuando Sevilla y Málaga. Barcelona es muy accesible respecto a los nudos localizados a lo largo de la costa Mediterránea, mientras su accesibilidad disminuye si se consideran las conexiones con los nudos de la costa septentrional.

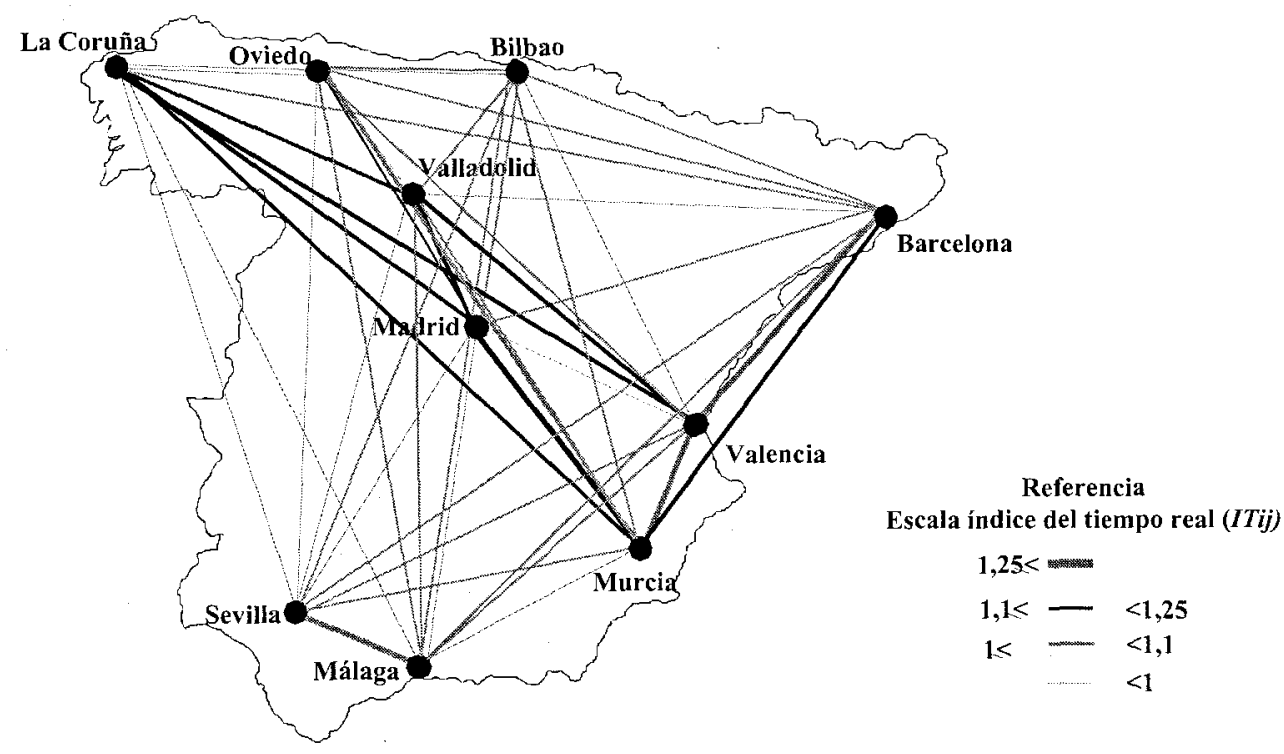

Figura 2. Índice del tiempo real $\left(I T_{i j}\right)$ en la España peninsular (1998).

La circulación por carretera de personas y bienes que se desarrolla a lo largo del mar Mediterráneo se beneficia de un óptimo grado de accesibilidad en el itinerario Barcelona-Valencia-Murcia, grado de accesibilidad que por otro lado disminuye en su prolongación hacia occidente. El grado de accesibilidad del tiempo real entre los nudos del arco atlántico (La Coruña, Oviedo y Bilbao) resulta en conjunto menos evolucionado debido al todavía incompleto desarrollo de la RGC. 


\subsection{La relación entre el desarrollo demográfico y la evolución del grado de accesibilidad}

Después de haber obtenido los datos del grado de accesibilidad se ha querido proponer una lectura de la relación entre accesibilidad y desarrollo demográfico. Tal propuesta se basa en la utilización de algunos instrumentos estadísticos de los cuales describiremos la finalidad especifica y los resultados alcanzados paso a paso. En las siguientes aplicaciones se considera:

- Número de habitantes: variable $x$

- Índice del tiempo real por cada nudo $i$ : variable $y$

En primer lugar la relación entre población y el grado de accesibilidad ha sido estudiada por medio del índice de cograduación calculado con los datos referidos al 1991 y al 1998 (Tabla 2). El índice de cograduación entre dos variables $x$ e $y$ no es otro que el coeficiente de correlación entre los rangos (o sea el número de orden) de las variables consideradas. Después de algunas simplificaciones, tal coeficiente puede expresarse del modo siguiente:

$$
r\left(v_{x}, v_{y}\right)=1-6 \sum\left(v_{x}-v_{y}\right)^{2} / N\left(N^{2}-1\right)
$$

De la fórmula se observa como la contribución al resultado del índice está dada exclusivamente por la diferencia $\left(v_{x}-v_{y}\right)^{2}$, o sea por la diferencia entre los rangos de las variables. El resultado del índice está comprendido entre -1 y 1.

Tabla 2. Rangos y diferencias entre los rangos de las variables población $(x)$ e índice de accesibilidad (y). Años 1991 y 1998.

\begin{tabular}{|c|c|c|c|c|c|c|c|c|}
\hline \multirow{2}{*}{ Nudos } & \multicolumn{5}{|c|}{ Año 1991} & \multicolumn{3}{|c|}{ Año 1998} \\
\hline & $V_{x}$ & $V_{y}$ & $V_{x}-V_{y}$ & $\left(V_{x}-V_{y}\right)^{2}$ & $V_{x}$ & $V_{y}$ & $V_{x}-V_{y}$ & $\left(V_{x}-V_{y}\right)^{2}$ \\
\hline Barcelona & 9 & 10 & -1 & 1 & 9 & 6 & 3 & 9 \\
\hline Bilbao & 5 & 2 & 3 & 9 & 5 & 3 & 2 & 4 \\
\hline Coruña (La) & 2 & 1 & 1 & 1 & 2 & 5 & -3 & 9 \\
\hline Madrid & 10 & 8 & 2 & 4 & 10 & 9 & 1 & 1 \\
\hline Málaga & 6 & 5 & 1 & 1 & 6 & 2 & 4 & 16 \\
\hline Murcia & 3 & 9 & -6 & 36 & 4 & 10 & -6 & 36 \\
\hline Oviedo & 1 & 4 & -3 & 9 & 1 & 4 & -3 & 9 \\
\hline Sevilla & 7 & 3 & 4 & 16 & 7 & 1 & 6 & 36 \\
\hline Valencia & 8 & 7 & 1 & 1 & 8 & 8 & 0 & 0 \\
\hline Valladolid & 4 & 6 & -2 & 4 & 3 & 7 & -4 & 16 \\
\hline
\end{tabular}

Fuente: Elaboración propria a partir de datos del Ministerio de Fomento. 
El valor del índice de cograduación disminuye pasando de 0'50 (1991) a 0’18 (1998). Esto indica que la relación entre población y accesibilidad disminuye en el intervalo de tiempo considerado. De hecho observando la tabla 2 se pueden evidenciar variaciones en el número de órden respecto al grado de accesibilidad para cada nudo considerado, excepto Oviedo, mientras el número de órden de cada nudo respecto a la población queda sustancialmente igual. Además es posible evidenciar que todos los nudos con un menor número de habitantes (La Coruña, Valladolid, Murcia, Bilbao) han tenido un aumento de su rango respecto al grado de accesibilidad. Refiriéndonos a los datos de 1998, los nudos que muestran una relación más débil entre las dos magnitudes consideradas son los nudos localizados en la vertiente occidental de la península; y el nudo de Murcia es aquel cuya desviación entre los rangos resulta, en términos absolutos, la más alta. En particular, el rango del índice de accesibilidad es más alto respecto al rango de población para los nudos de Oviedo, La Coruña, Valladolid y Murcia, mientras que en el caso opuesto están los nudos de Sevilla y Málaga. Un mayor equilibrio entre población y accesibilidad se registra en los nudos de la vertiente oriental de la península, comprendiendo también Madrid.

Es importante resaltar que el aumento de la accesibilidad de los nudos considerados ha. tendido a homogeneizarse. Así se puede probar directamente a través de la disminución de los valores $\sigma(y)$ y c.v.(y) en el año 1998 respecto a los valores de 1991 (Tabla 3).

Tabla 3. Algunos valores relativos al coeficiente de correlación lineal y a la recta de regresión lineal. Años 1991 y 1998.

\begin{tabular}{|cccc|}
\hline Estadisticas descriptivas & Magnitud & Año 1991 & Año 1998 \\
& $m(x)$ & 5,7352 & 5,7364 \\
& $m(y)$ & 0,8532 & 1,0401 \\
$\sigma(y)$ & 0,8094 & 0,7883 \\
& $\sigma(y)$ & 0,0606 & 0,0302 \\
& $c . v \cdot(x)$ & 0,1411 & 0,1374 \\
& $c . v \cdot(y)$ & 0,0710 & 0,0290 \\
$\operatorname{cov}(x, y)$ & 0,0284 & 0,0111 \\
& $n(x, y)$ & 0,5796 & 0,4663 \\
& $m\left(\log _{10} x\right)$ & 5,7354 & 5,7364 \\
& $\sigma\left(\log _{10} x\right)$ & 0,3511 & 0,3442 \\
& $\operatorname{cov}\left(\log _{10} x, y\right)$ & 0,0134 & 0,0042 \\
\hline Regresión lineal & $a$ & 0,2312 & 0,8370 \\
& $b$ & 0,1087 & 0,0354 \\
& $r\left(\log _{10} x, y\right)$ & 0,6285 & 0,4012 \\
\hline
\end{tabular}

$m$, indica la media

$\sigma$, indica la desviación cuadrática media

c.v., indica el coeficiente de variación

cov, indica la covariancia

$r$, indica el coeficiente de correlación

$a$, indica la intercepción de la recta de regresión lineal

$b$, indica el coeficiente angular de la recta de regresión lineal.

Fuente: Elaboración propia a partir de datos del Ministerio de Fomento. 
Como siguiente medida de la relación existente entre población y grado de accesibilidad se ha utilizado el coeficiente de correlación lineal $(r(x, y))$ entre población e índice de accesibilidad. Los resultados, recogidos en la tabla 3 , sugieren una correlación positiva entre las dos magnitudes $(r(x, y)$ es positivo): con el aumento de la población del nudo aumenta también su grado de accesibilidad aunque tal relación resulta menos fuerte en 1998 ya que su valor es menor al de 1991.

Por otro lado se ha analizado la relación objeto de estudio describiendo en qué términos se mueve el grado de accesibilidad dependiendo de la variación de la población y utilizando el instrumento de regresión lineal (Figura 3 A-B y Tabla 3). La población ha sido considerada como variable independiente y el grado de accesibilidad como variable dependiente. Ya que los nudos de Barcelona y Madrid provocan, dado el elevado número de habitantes respecto a los otros ocho nudos de la red-sistema, un efecto potencialmente desviado en el estudio de la relación existente entre las dos magnitudes, se ha creído preferible expresar la población en el en el modo de obtener "redimensionamiento" homogéneo del número de habitantes para todos los nudos.

Los resultados que se obtienen de esta aplicación evidencian una ligazón directa entre población y accesibilidad (coeficiente $b$ positivo: al aumentar $x$ aumenta también y). En todo caso esta ligazón se debilita desde 1991 a 1998: de hecho $b$ pasa de 0'1087 a 0'0354; lo que viene a confirmar el resultado de mayor homogeneidad del grado de accesibilidad en los puntos del territorio considerados: no sólo las ciudades más pobladas tienen un grado de accesibilidad más alto sino que la accesibilidad tiende a aumentar también para las ciudades menos pobladas.

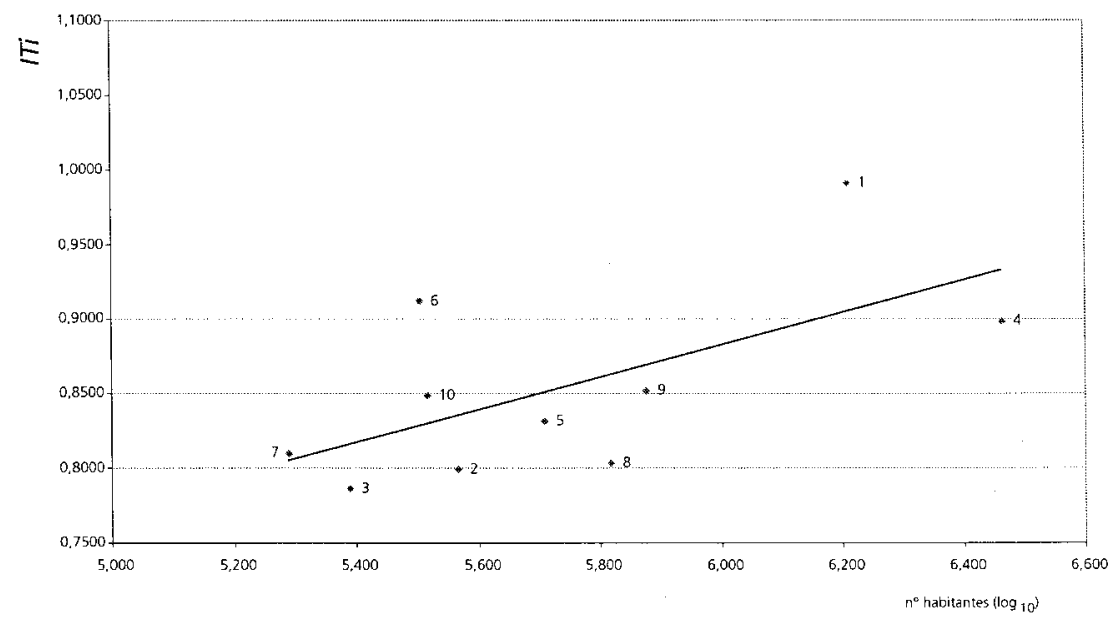

Figura 3A. Función de regresión linear entre $T_{i}$ y $n .^{\circ}$ de habitantes $\left(\log _{10}\right)$ año 1991. 


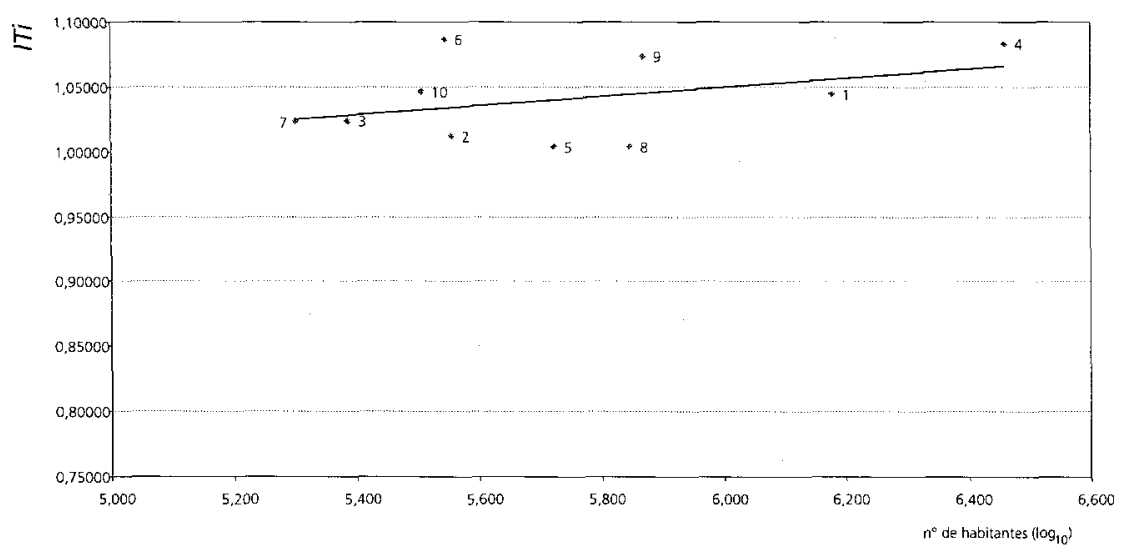

Figura 3B. Función de regresión lineal entre $1 T_{i}$ y n. ${ }^{\circ}$ de habitantes $\left(\log _{10}\right)$ año 1998.

La recta de regresión lineal indica sintéticamente tales resultados; en referencia a la primera afirmación (que la relación entre las dos magnitudes se debilita en los dos años tomados en consideración), se nota cómo la inclinación de la recta es positiva ( $b$ positivo) pero su inclinación es menos marcada de 1998 respecto a la de 1991. Además, si se confrontan las gráficas de dichos años se nota que todos los nudos "salen" en el plano cartesiano relativo a 1998 significando un mayor grado de accesibilidad alcanzado por los mismos nudos.

\section{Conclusiones}

En este artículo se analiza la relación entre infraestructura de transporte (carreteras) y desarrollo regional, y en particular entre la accesibilidad viaria y desarrollo demográfico. En un primer momento se ha querido ofrecer una visión general sobre la red viaria española después de las intervenciones sucesivas durante la segunda mitad de los años ochenta. Seguidamente se ha propuesto una aplicación metodológica que tiende a interpretar la relación mencionada.

El método utilizado se ha mostrado adecuado para probar que todos los nudos dispersos en el territorio han incrementado su grado de accesibilidad y que existe una ligazón entre las dos magnitudes: accesibilidad y desarrollo demográfico. A la luz de tales resultados es posible afirmar que las obras de ampliación y modernización de la red viaria española comprendidas dentro del Plan de Carreteras 1984-91 y sucesivamente inter- 
caladas en el Plan de las Infraestructuras 1993-2007 han incrementando la accesibilidad de los nudos escogidos para el examen y también han homogeneizado tal magnitud en los mismos. Esta última observación se evidencia a través de la disminución de los valores del $\sigma(y)$ y del c.v.(y) en 1998 respecto a 1991 y a través del instrumento de la regresión lineal que, en su aplicación gráfica, reasume muchos de los resultados obtenidos.

\section{Notas}

* Este trabajo representa la síntesis de una parte de la tesis para la licenciatura en Geografía Económica, presentada en la Facultad de Economía de Florencia.

1 Modelo de accesibilidad potencial realizado bajo la supervisión del Ing. Pedro Galán Bueno (Jefe del Servicio de Proyecto de la Dirección General de Carreteras).

2 Oficialmente está considerada como carretera "el recorrido de dominio y uso público proyectado y construido fundamentalmente para la circulación de automóviles" (Ministerio de Fomento, Secretaría General Técnica, 1999:7).

3 Estos ejes están representados por seis autopistas; desde el Norte, en sentido de las agujas del reloj tenemos: la NI Madrid-San Sebastian-Irún (Autovía del Norte); la NII Madrid-Barcelona-La Junquera (Autovía de Aragón); la NII Madrid-Valencia (Autovía de Levante); la NIV Madrid-Cordoba-Sevilla-Cádiz (Autovía de Andalucía); la NV Madrid-Badajoz (Autovía de Extremadura); la NVI Madrid-La Conña (Autovía del Noroeste).

4 La distancia entre $i-j$ en términos kilométricos es directamente determinable, mientras que el tiempo de recorrido entre $i$-j surge indirectamente de la base de la relación: $T_{i j}=D_{i j} / V_{i j}$, recurriendo a algunas adquisiciones en base a la velocidad de circulación de $i$ a $j$.

5 La técnica del vehículo flotante permite definir la velocidad media y teórica de circulación de un automóvil de uso privado en la red de carreteras. La velocidad del vehículo va en función a las condiciones técnicas del trazado (planialtrimetría, estado del trazado, tortuosidad, etc.) y de las condiciones del tráfico. El vehículo en cuestión simula en el comportamiento del flujo de la circulación y adelantará tantos vehículos como le sobrepasen manteniendo un régimen de circulación de seguridad. La velocidad del "vehículo flotante" será la velocidad media considerando los diversos estados de tráfico (sin tráfico, tráfico normal o tráfico congestionado) y los aspectos ya señalados.

6 Podemos añadir que la relación $D_{i j}^{\prime} / D_{i j}$ es siempre $\leq 1$ mientras $V_{i f} \tilde{V}$ depende de la velocidad media sobre el tramo $i-j\left(V_{i j}\right)$ que obviamente puede ser mayor o menor de la velocidad media sobre toda la red $\left(\tilde{V}\right.$. Sobre esta base observamos que siempre: $I D_{i j} \leq 1$ mientras $I T_{i j}$ puede ser también superior a 1 en el caso en que la velocidad $\tilde{V}_{i j}$ sea significativamente mayor de la media $\tilde{V}$.

7 Obviamente se pueden construir índices en los cuales cada tiempo está ponderado en función de la importancia, según un criterio concreto, del nudo de liegacla. De todos modos, un enfoque de este genero presenta a nuestro parecer algunos incovenientes: una cierta arbitrariedad en la selección de la variable del peso; el hecho de que selecciones diversas de tal variable pueden levar a conclusiones divergentes; la disminución de las ventajas antes consideradas en términos de simplicidad y facilidad de la interpretación.

8 El número de habitantes de cada nudo (ciudad) se refiere al territorio municipal, según los datos disponibles en los archivos del INE. Han sido considerados los habitantes del municipio en vez de aquéllos de las áreas metropolitanas, dado que el territorio municipal tiene una delimitación univoca sobre la base de sus límites administrativos, mientras la clelimitación territorial de las áreas metropolitanas varía en relación a las distintas teorías consideradas. En España el término "área metropolitana" aparece por primera vez en la legislación de 1963 (Plan General de Ordenación Urbana de Madrid), pero todavía no existe una definición oficial para área metropolitana (Bielza de Ory et al., 1993: 92-93). 


\section{Bibliografía}

AA.VV. (1990): Jornada sobre las carreteras, Actas. Vitoria-Gasteiz.

Biehl, D. (1986): The contribution of Infrastructure to the Regional development $\mathrm{n}^{\circ}$. 1-2. Luxemburgo, Oficina de publicaciones oficiales de las Comunidades Europeas.

Biehl, D. (1988): Las infraestructuras y el desarrollo regional. Papeles de Economia Española, 35, p. 293-310.

Bielza de Ory, V. et al. (1993): Geografía General. Madrid, Taurus, tomo 2.

EEC (1989): Council resolution on the transeuropean network. Bruselas. COM (89) 643.

ECC (1995): Final report on the guidelines for the trans-European airport network. Bruselas.

Capineri, C., Tinacci M. et al. (1996): Geografia delle comunicazioni: reti e strutture territoriali. Turín, Giappichelli.

Gutiérrez Puebla, J. y Monzón de Cáceres, A. (1993): La accesibilidad a los centros de actividad económica antes y después de Plan Director de Infraestructuras. Ciudad $y$ Territorio, 97, p. 385-401.

Gutiérrez Puebla, J. (1998): Redes, espacios y tiempo. Anales de geografía de la Universidad de Complutense, 18, p. 60-76.

INE (Varios años): Series Estadisticas.

Ministerio de Fomento (1999): Los transportes $y$ las comunicaciones, Informe anual 1998. Madrid.

Ministerio de Fomento (1999): Leyes de carreteras y Autopistas. ( $3^{\mathrm{a}}$ ed.). Madrid.
Ministerio de Fomento (1999): Mapa de tráfico 1998. Madrid.

Ministerio de Fomento (1999): Mapa de velocidades 1998. Madrid.

Ministerio de Fomento (1999): Mapa oficial de carreteras 1998. Madrid.

Ministerio de Fomento (1999): Los transportes $y$ las comunicaciones, Informe anual 1998. Madrid.

Ministerio de Fomento (1995): Inventario red de carreteras. Madrid

MOPU (1984): Los Transportes y las comunicaciones, Informe anual 1984. Madrid.

MOPU (1984): Plan General de Carreteras 1984/91. vol. 8-I. Madrid, Dirección General de Carreteras.

MOPU (1993): El Plan General de carreteras, realizaciones. Madrid.

MOPU (1994): Plan General de carreteras, balance. Madrid.

MOPU (1994): Plan Director de Infraestructuras 1993-2007. Madrid.

Monzón de Cáceres, A. (1988): Los indicadores de accesibilidad y la planificación de transporte: concepto y clasificación. Transporte Turismo y Comunicaciones, 35, p. 11-18.

Moreno Jiménez, A. (1985): Metodos para el estudio de la estructura y la organización de los sistemas espacio-funcionales. Estudios territoriales, 17, p. 123-144.

Nice, B. (1976): Il ruolo delle autostrade nell'organizzazione territoriale in Italia. Italian Contributions $23^{\text {rd }}$ Int. Geog. Congress 1976. Roma. 
Peréz Esparcia, J. (1988): Infraestructuras de transporte y su relaciones con los cambios demográficos y el desarollo económico de España (1960/85). Estudios Geográficos, p. 381-398.

Puncel Chornet, A. (1996): La autopista del mediterráneo. Valencia, Servicio de Publicaciones de la Universidad de Valencia.

Sáenz de Buruaga, G. (1989): Dinámica y ejes de desarrollo en España. Colegio de Madrid, VI, 35, p. 288-293.
Santos, M. (1996): Los nuevos mundos de la Geografía. Anales de Geografía de la Universidad Complutense, 16, p. 15-27.

Santos, M. (1993): Los espacios de la globalización. Anales de Geografía de la Universidad Complutense, 13, p. 69-77.

Turró Calvet, M. (1996): La accesibilidad, criterio básico para el desarollo de un sistema de transporte eficaz, sostenible y choesivo. Obras públicas, 35, $\mathrm{p}$. 18-27. 